\title{
EXAMPLES OF INFINITY AND LIE ALGEBRAS AND THEIR VERSAL DEFORMATIONS
}

\author{
ALICE FIALOWSKI \\ Department of Applied Analysis, Eötvös Loránd University \\ Pázmány Péter sétány $1 C, H-1117$ Budapest, Hungary \\ E-mail: fialowsk@cs.elte.hu \\ MICHAEL PENKAVA \\ Department of Mathematics, University of Wisconsin, Eau Claire, WI 54702-4004, U.S.A. \\ E-mail: penkavmr@uwec.edu
}

\begin{abstract}
This article explores some simple examples of $L_{\infty}$ algebras and the construction of miniversal deformations of these structures. Among other things, it is shown that there are two families of nonequivalent $L_{\infty}$ structures on a $1 \mid 1$ dimensional vector space, two of which are Lie algebra structures. The main purpose of this work is to provide a simple effective procedure for constructing miniversal deformations, using the examples to illustrate the general technique. The same method can be applied directly to construct versal deformations of Lie algebras.
\end{abstract}

1. Introduction. Global and local deformations of different algebraic objects are in the center of current research. We just mention the Krichever-Novikov algebras as deformations of the Virasoro algebra [12], string theory [1], as well as the recent application of deformation theory to solve the problem of quantization of Poisson manifolds [11].

In this paper we consider local (formal) deformations. There is much confusion in the literature if one wants to solve the deformation question completely - although this is the main goal in deformation theory. We search for a "universal" object in the category of deformations. Such an object usually does not exist, but there exist a so called "versal" or "miniversal" element. Intuitively, this versal element induces all nonequivalent deformations and is unique at the infinitesimal level.

2000 Mathematics Subject Classification: 14D15, 13D10, 14B12, 16S80, 16E40, 17B55, 17B70.

Key words and phrases: versal deformations, infinity algebras, Lie algebras, Lie superalgebras.

The research of the authors was partially supported by grants OTKA T030823, T29525, FKFP 0170/1999 and by grants from the University of Wisconsin-Eau Claire.

Received 13 January 2001; revised 16 November 2001.

The paper is in final form and no version of it will be published elsewhere. 
There are some known interesting results for constructing versal deformations of Lie algebras (see [3], [6]). A natural generalization leads to strongly homotopy Lie algebras (sometimes called $L_{\infty}$ algebras or sh Lie algebras) This appears for instance in closed string field theory (see [22], or in the study on higher spin particles (see [3], but also gives rise to cycles in the homology of graph complexes as described first by Kontsevich (see [9]). Although sh Lie algebras arise in many different aspects, so far there were no explicit examples, nor was their deformation theory studied.

In this article we shall explore some interesting simple examples of $L_{\infty}$ structures on low dimensional $\mathbb{Z}_{2}$-graded vector spaces, and construct versal deformations of these structures. A detailed explanation of the definitions of infinity algebras and their cohomology theory can be obtained in $[16,17]$. Infinity algebras were first described and applied by James Stasheff (see $[23,24,14,13]$ ), and have appeared recently in both mathematics and mathematical physics (see $[1,2,10,15,25,20,19,18]$ ). The essential details of the theory of versal deformations of infinity algebras are outlined in [8], and are a generalization of the results in [7] on versal deformations of Lie algebras (see also [4, 5, 6, 9, 21]). In this article, our aim is to show how to construct a miniversal deformation through some examples. For the proof of the miniversality of the deformations we will construct here, we refer to [8]. For sake of completeness, we will present here the basic definitions of $L_{\infty}$ algebras and formal deformations.

\section{Basic definitions}

2.1. $L_{\infty}$ algebras. If $W$ is a $\mathbb{Z}_{2}$-graded vector space, then $S(W)$ denotes the symmetric coalgebra of $W$. If we let $T(W)$ be the reduced tensor algebra $T(W)=\bigoplus_{n=1}^{\infty} W^{\otimes n}$, then the reduced symmetric algebra $S(W)$ is the quotient of the tensor algebra by the graded ideal generated by $u \otimes v-(-1)^{u v} v \otimes u$ for elements $u, v \in W$. The symmetric algebra has a natural coalgebra structure, given by

$$
\Delta\left(w_{1} \ldots w_{n}\right)=\sum_{k=1}^{n} \sum_{\sigma \in \operatorname{Sh}(k, n-k)} \epsilon(\sigma) w_{\sigma(1)} \ldots w_{\sigma(k)} \otimes w_{\sigma(k+1)} \ldots w_{\sigma(n)},
$$

where we denote the product in $S(W)$ by juxtaposition, $\operatorname{Sh}(k, n-k)$ is the set of unshuffles of type $(k, n-k)$, and $\epsilon(\sigma)$ is a sign determined by $\sigma$ (and $\left.w_{1} \ldots w_{n}\right)$ given by

$$
w_{\sigma(1)} \ldots w_{\sigma(n)}=\epsilon(\sigma) w_{1} \ldots w_{n} .
$$

A coderivation on $S(W)$ is a map $\delta: S(W) \rightarrow S(W)$ satisfying

$$
\Delta \circ \delta=(\delta \otimes I+I \otimes \delta) \circ \Delta .
$$

Let us suppose that the even part of $W$ has basis $e_{1} \ldots e_{m}$, and the odd part has basis $f_{1} \ldots f_{n}$, so that $W$ is an $m \mid n$ dimensional space. Then a basis of $S(W)$ is given by all vectors of the form $e_{1}^{k_{1}} \ldots e_{m}^{k_{m}} f_{1}^{l_{1}} \ldots f_{n}^{l_{n}}$, where $k_{i}$ is any nonnegative integer, and $l_{i} \in \mathbb{Z}_{2}$. An $L_{\infty}$ structure on $W$ is simply an odd codifferential on $S(W)$, that is to say, an odd coderivation whose square is zero. The space $\operatorname{Coder}(W)$ can be naturally identified with $\operatorname{Hom}(S(W), W)$, and the Lie superalgebra structure on $\operatorname{Coder}(W)$ determines a Lie bracket on $\operatorname{Hom}(S(W), W)$ as follows. Denote $L_{m}=\operatorname{Hom}\left(S^{m}(W), W\right)$ so that $L=$ $\operatorname{Hom}(S(W), W)$ is the direct product of the spaces $L_{i}$. If $\alpha \in L_{m}$ and $\beta \in L_{n}$, then $[\alpha, \beta]$ 
is the element in $L_{m+n-1}$ determined by

$$
\begin{aligned}
{[\alpha, \beta] } & \left(w_{1} \ldots w_{m+n-1}\right) \\
= & \sum_{\sigma \in \operatorname{Sh}(n, m-1)} \epsilon(\sigma) \alpha\left(\beta\left(w_{\sigma(1)} \ldots w_{\sigma(n)}\right) w_{\sigma(n+1)} \ldots w_{\sigma(m+n-1)}\right) \\
& -(-1)^{\alpha \beta} \sum_{\sigma \in \operatorname{Sh}(m, n-1)} \epsilon(\sigma) \beta\left(\alpha\left(w_{\sigma(1)} \ldots w_{\sigma(m)}\right) w_{\sigma(m+1)} \ldots w_{\sigma(m+n-1)}\right) .
\end{aligned}
$$

Another way to express this bracket is in the form

$$
[\alpha, \beta]=\alpha \tilde{\beta}-(-1)^{\alpha \beta} \beta \tilde{\alpha},
$$

where for $\varphi \in \operatorname{Hom}\left(S^{k}(W), W\right), \tilde{\varphi}$ is the associated coderivation, given by

$$
\tilde{\varphi}\left(w_{1} \ldots w_{n}\right)=\sum_{\sigma \in \operatorname{Sh}(k, n-k)} \epsilon(\sigma) \varphi\left(w_{\sigma(1)} \ldots w_{\sigma(k)}\right) w_{\sigma(k+1)} \ldots w_{\sigma(n)} .
$$

In this article, formula (1) will be used extensively to make explicit computations of brackets. In our computations, we shall also use the fact that there are $\left(\begin{array}{l}n \\ k\end{array}\right)$ permutations in $\operatorname{Sh}(k, n-k)$.

If $W$ is completely odd, and $d \in L_{2}$, then $d$ determines an ordinary Lie algebra on $W$, or rather on its parity reversion. The symmetric algebra on $W$ looks like the exterior algebra on $W$ if we forget the grading. If we define $[a, b]=d(a b)$ for $a, b \in W$, then the bracket is antisymmetric because $b a=-a b$, and moreover

$$
\begin{aligned}
0=[d, d](a b c) & =\frac{1}{2} \sum_{\sigma \in \operatorname{Sh}(2,1)} \epsilon(\sigma) d(d(\sigma(a) \sigma(b)) \sigma(c))=d((d(a b) c)+d(d(b c) a)-d(d(a c) b) \\
& =[[a, b], c]+[[b, c], a]-[[a, c], b],
\end{aligned}
$$

which is the Jacobi identity. When $d \in L_{2}$ and $W$ has a true grading, then the same principle holds, except that one has to take into account a sign arising from the map $S^{2}(W) \rightarrow \bigwedge^{2}(V)$, where $V$ is the parity reversion of $W$. Thus $\mathbb{Z}_{2}$-graded Lie algebras are also examples of $L_{\infty}$ algebras. In addition, differential graded Lie algebras are examples of $L_{\infty}$ algebras. In all these cases, the method of construction of miniversal deformations we describe here applies. One simply considers only terms that come from $L_{2}$ in the codifferentials.

Suppose that $\tilde{g}: S(W) \rightarrow S\left(W^{\prime}\right)$ is a coalgebra morphism, that is a map satisfying

$$
\Delta^{\prime} \circ \tilde{g}=(\tilde{g} \otimes \tilde{g}) \circ \Delta .
$$

If $d$ and $d^{\prime}$ are $L_{\infty}$ algebra structures on $W$ and $W^{\prime}$, resp., then $\tilde{g}$ is a homomorphism between these structures if $\tilde{g} \circ d=d^{\prime} \circ \tilde{g}$. Two $L_{\infty}$ structures $d$ and $d^{\prime}$ on $W$ are equivalent when there is a coalgebra automorphism $\tilde{g}$ of $S(W)$ such that $d^{\prime}=\tilde{g}^{-1} \circ d \circ \tilde{g}$. Furthermore, if $d=d^{\prime}$, then $\tilde{g}$ is said to be an automorphism of the $L_{\infty}$ algebra.

2.2. Deformations. We shall always assume that the ground field $\mathfrak{K}$ of the graded space $W$ has characteristic zero. A local base is a $\mathbb{Z}_{2}$-graded commutative local algebra $\mathcal{A}$ equipped with a fixed augmentation $\epsilon: \mathcal{A} \rightarrow \mathfrak{K}$, with augmentation ideal $\mathfrak{m}$.

Definition 2.1. If $d$ is a codifferential on $S(W)$, then a deformation of $d$ with base $\mathcal{A}$ is a codifferential $d_{\mathcal{A}}$ on $S(W) \otimes \mathcal{A}$, which projects to $d$ under the morphism of coalgebras 
$I \otimes \epsilon: S(W) \otimes \mathcal{A} \rightarrow S(W)$. When $\mathcal{A}$ is a formal algebra (a complete local algebra), then a deformation with base $\mathcal{A}$ will be called a formal deformation, and a deformation with base $\mathcal{A} / \mathfrak{m}^{n+1}$ will be called an $n$-th order deformation. When $n=1$, the deformation is called infinitesimal.

Definition 2.2. A deformation $d_{\mathcal{A}}$ with local base $\mathcal{A}$ is called versal or miniversal if for any deformation $d_{\mathcal{A}}^{\prime}$ with base $\mathcal{A}^{\prime}$, there is a certain morphism of algebras $\lambda: \mathcal{A} \rightarrow \mathcal{A}^{\prime}$, such that the induced codifferential $\lambda_{*}\left(d_{\mathcal{A}}\right)$ on $S(W) \otimes \mathcal{A}^{\prime}$ is equivalent to $d_{\mathcal{A}}^{\prime}$.

In [8], it was shown that under some mild restrictions on the cohomology (it must be of finite type), there is a miniversal deformation, Note that any coderivation of $S(W) \otimes \mathcal{A}$ lies in $L \otimes \mathcal{A}$, so that $\lambda_{*}$ is simply the natural map $L \otimes \mathcal{A} \rightarrow L \otimes \mathcal{A}^{\prime}$. Moreover, the notion of equivalence of deformations with base $\mathcal{A}$ is given by the following. If $d$ is a codifferential on $S(W)$ and $\tilde{g}$ is a coalgebra automorphism of $S(W)$, then define $\tilde{g}^{*}(d)=\tilde{g}^{-1} \circ d \circ \tilde{g}$. If $d_{\mathcal{A}}^{1}$ and $d_{\mathcal{A}}^{2}$ are two deformations of $d$ with base $\mathcal{A}$, then they are said to be equivalent if there is a coalgebra automorphism $\tilde{g}$ of $S(W) \otimes \mathcal{A}$ satisfying $(I \otimes \epsilon) \circ \tilde{g}=I \otimes \epsilon$ such that $\tilde{g}^{*}\left(d_{\mathcal{A}}^{1}\right)=d_{\mathcal{A}}^{2}$. The condition on $\tilde{g}$ means simply that $\tilde{g}(w)=w \quad(\bmod \mathfrak{m})$, so that $\tilde{g}$ does not act on the underlying coalgebra $S(W)$ (to avoid mixing the notion of automorphisms of the $L_{\infty}$ algebra with that of the equivalences of deformations of the algebra).

The objective in this article is to compute versal deformations of some simple examples. To see how to do this in practice, we will give a concrete description of the construction of a miniversal deformation of a codifferential $d$. Since $d$ is a codifferential, the map $D: L \rightarrow L$ given by $D(\varphi)=[d, \varphi]$ satisfies $D^{2}=0$. The homology of this map is called the cohomology of the $L_{\infty}$ algebra. Let us assume that the space of cocycles $Z(L)$ has an increasing basis of the form $\left\langle\delta_{i}, \beta_{j}\right\rangle$, where the subspace spanned by the $\delta_{i}$ projects isomorphically onto the cohomology of $d$, and $\beta_{j}$ is a basis of the coboundaries. By basis, we mean (in the sense of [8]) that any element of $Z(L)$ has a unique expression as a power series in the elements of the basis, and increasing means that the orders of the elements are nondecreasing. Here, order refers to the order of the element in $L$. In the general case, an element in $L$ is represented as a power series, since $L$ is a direct product of the spaces $L_{n}$. The order represents the least $n$ such that the projection of the element onto $L_{n}$ is nonzero.

In order for there to be a good theory of deformations, the cohomology should be of finite type, which means that there are only a finite number of elements in the basis $\delta_{i}$ of order less than a given integer. In our examples, the condition will be satisfied, but even in the simplest cases, as we shall see in the case of a one dimensional space, the cohomology can be infinite dimensional.

To construct the universal infinitesimal deformation of a codifferential $d$, one formally considers the infinitesimal base $\mathcal{A}=\mathfrak{K} \oplus \mathcal{H}$ where $\mathcal{H}=(\Pi(H(L)))^{*}$ is the dual of the parity reversion of the cohomology of $L$, and the multiplication in $\mathcal{H}$ is trivial. Then the base of the miniversal deformation is $\mathcal{A}=\mathfrak{K}[[\mathcal{H}]] / R$, where $R$ represents a set of polynomial relations involving the generators of the space $\mathcal{H}$.

If we let $\delta_{i}$ be a basis of a subspace of cocycles which projects isomorphically to the cohomology, then choosing an appropriate dual basis $u^{i}$ of $\mathcal{H}$, the universal infinitesimal deformation $d_{1}$ is given by 


$$
d_{1}=d+\delta_{i} u^{i} .
$$

The parity of the element $u^{i}$ is opposite to the parity of $\delta_{i}$, in order to preserve the oddness of the codifferential. Let $\alpha_{1}=\delta_{i} u^{i}$. Then $\alpha_{1}$ is a cocycle. In order to extend the bracket to a second order bracket, one simply computes

$$
\left[d_{1}, d_{1}\right]=[d, d]+2\left[d, \alpha_{1}\right]+\left[\alpha_{1}, \alpha_{1}\right]=\left[\alpha_{1}, \alpha_{1}\right]
$$

which is a cocycle, because the bracket of two cocycles is always a cocycle. This cocycle can be represented as a linear combination of the $\delta_{i}$ plus a coboundary term. Let us express this in the form

$$
\left[d_{1}, d_{1}\right]=-\frac{1}{2} D\left(\alpha_{2}\right)+\delta_{i} R_{2}^{i},
$$

where $R_{2}^{i}$ is a sum of products of the parameters of the form $u_{k} u_{l}$, so lies in $\mathfrak{m}^{2}$, and $\alpha_{2} \in L \otimes \mathfrak{m}^{2}$. We would like $\left[d_{1}, d_{1}\right]$ to be a coboundary mod $\mathfrak{m}^{3}$, which means that we must have $R_{2}^{i}=0 \bmod \mathfrak{m}^{3}$.

The second order deformation is given by $d_{2}=d_{1}+\alpha_{2}$. The bracket of this second order codifferential with itself is

$$
\begin{aligned}
{\left[d_{2}, d_{2}\right] } & =\left[d_{1}, d_{1}\right]+2\left[d_{1}, \alpha_{2}\right]+\left[\alpha_{2}, \alpha_{2}\right]=\left[d_{1}, d_{1}\right]+2 D\left(\alpha_{2}\right)+2\left[\alpha_{1}, \alpha_{2}\right]+\left[\alpha_{2}, \alpha_{2}\right] \\
& =\delta_{i} R_{2}^{i}+2\left[\alpha_{1}, \alpha_{2}\right]+\left[\alpha_{2}, \alpha_{2}\right]
\end{aligned}
$$

Note that the first and third terms in this bracket are zero mod $\mathrm{m}^{3}$. We want this bracket to be a coboundary mod $\mathfrak{m}^{3}$. Let us first show that at least it is a cocycle mod $\mathfrak{m}^{3}$. Now

$$
\begin{aligned}
D\left(\left[d_{2}, d_{2}\right]\right) & =2\left[\alpha_{1}, D\left(\alpha_{2}\right)\right]+2\left[D\left(\alpha_{2}\right), \alpha_{2}\right]=2\left[\alpha_{1},-2\left[\alpha_{1}, \alpha_{1}\right]+2 \delta_{i} R_{2}^{i}\right]+2\left[D\left(\alpha_{2}\right), \alpha_{2}\right] \\
& =4\left[\alpha_{1}, \delta_{i} R_{2}^{i}\right]+2\left[D\left(\alpha_{2}\right), \alpha_{2}\right],
\end{aligned}
$$

which is equal to zero mod $\mathfrak{m}^{4}$. In general, let us suppose that we have shown that $\left[d_{n}, d_{n}\right]=0 \bmod \mathfrak{m}^{n+1}$. and that $D\left(\left[d_{n}, d_{n}\right]\right)=0 \bmod \mathfrak{m}^{n+2}$. Then we set

$$
\left[d_{n}, d_{n}\right]=-\frac{1}{2} D\left(\alpha_{n+1}\right)+\delta_{i} R_{n+1}^{i}
$$

where $\alpha_{n+1} \in L \otimes \mathfrak{m}^{n+1}$, and $R_{n+1}^{i}=0 \bmod \mathfrak{m}^{n+2}$. Set $d_{n+1}=d_{n}+\alpha_{n+1}$. Then

$$
\left[d_{n+1}, d_{n+1}\right]=\delta_{i} R_{n+1}^{i}+2\left[d_{n}-d, \alpha_{n+1}\right]+\left[\alpha_{n+2}, \alpha_{n+2}\right] .
$$

Note that each term appearing in the bracket is equal to zero $\bmod \mathfrak{m}^{n+2}$. Next, we compute

$$
\begin{aligned}
D\left[d_{n+1}, d_{n+1}\right] & =2\left[D\left(d_{n}-d\right), \alpha_{n+1}\right]-2\left[d_{n}-d, D \alpha_{n+1}\right]+2\left[\alpha_{n+1}, \alpha_{n+1}\right] \\
& =2\left[D\left(d_{n}-d-\alpha_{1}\right), \alpha_{n+1}\right]-2\left[d_{n}, D \alpha_{n+1}\right]+2\left[\alpha_{n+1}, \alpha_{n+1}\right] \\
& =2\left[D\left(d_{n}-d-\alpha_{1}\right), \alpha_{n+1}\right]-2\left[d_{n},-2\left[d_{n}, d_{n}\right]+2 \delta_{i} R_{n+1}^{i}\right]+2\left[\alpha_{n+1}, \alpha_{n+1}\right] \\
& =2\left[D\left(d_{n}-d-\alpha_{1}\right), \alpha_{n+1}\right]-2\left[d_{n}, 2 \delta_{i} R_{n+1}^{i},+\right] 2\left[\alpha_{n+1}, \alpha_{n+1}\right] \\
& =2\left[D\left(d_{n}-d-\alpha_{1}\right), \alpha_{n+1}\right]-2\left[d_{n}-d, 2 \delta_{i} R_{n+1}^{i}\right]+2\left[\alpha_{n+1}, \alpha_{n+1}\right] .
\end{aligned}
$$

Each of the three remaining terms is equal to zero $\bmod \mathfrak{m}^{n+3}$, so one can continue the process by adding an $\alpha_{n+2}$ term. Thus we see that it is possible to construct a formal deformation $d_{\infty}=\sum_{i=1}^{\infty} \alpha_{i}$, which will satisfy $\left[d_{\infty}, d_{\infty}\right]=0$. But what about the relations $R_{n}^{i}$ ? It is easy to see that $R_{n+1}^{i}=R_{n}^{i} \bmod \mathfrak{m}^{n+1}$. We obtain a series of relations $R_{\infty}^{i}$, which must hold on the base in order for the required bracket relation to hold. 
When it happens that $\left[d_{n}, d_{n}\right]$ contains no coboundary terms for some $n$, then the $n$-th order deformation is miniversal, and the $n$-th order relations hold to all orders. Thus, in good cases, one may hope to calculate the miniversal deformation exactly. This is what happens in the examples we shall study.

We shall only consider the simplest cases of $L_{\infty}$ algebras. It is interesting to note that even for a 2 dimensional vector space, it can happen that there are infinitely many nonequivalent $L_{\infty}$ structures. The main purpose of this article is to demonstrate how to compute versal deformations in practice.

Note that in order to calculate a versal deformation, we need much more information than the dimensions of the cohomology groups. Thus, calculations of cohomology of Lie algebras which often appear in the literature are not concrete enough to do versal deformations, because the bracket structure on the cohomology is essential for the calculations, because only the dimensions of the cohomology groups are computed. In fact in the examples presented in this paper, we had to also compute brackets of cochains that are not cocycles.

3. Deformations of a $0 \mid 1$ dimensional space. Suppose that $W=\langle f\rangle$ is a 1 dimensional odd vector space. This situation corresponds by parity reversion to the case of an ordinary even one dimensional space, on which there is obviously only the trivial Lie algebra structure. Indeed, there is only one $L_{\infty}$ algebra structure $d=0$ as well, so it does not seem reasonable to expect any deformations of this trivial structure. Nevertheless, this intuition is slightly wrong, because there is a infinitesimal differential on the space $W$, and a differential graded space is an $L_{\infty}$ algebra. To see what is going on, note that $L_{1}=\langle\varphi\rangle$, where $\varphi(f)=f$ is the identity map on $W$, which is an even 1-cochain. The map $\varphi$ does not determine an $L_{\infty}$ structure, because it is even, but when multiplied by the odd parameter $\theta$, it becomes an odd codifferential on $S(W)=W$. Thus $d_{1}=\varphi \theta$ is an infinitesimal deformation of the trivial deformation $d=0$. Since $\varphi$ is a cocycle, but not a coboundary, this deformation is also a miniversal deformation. To determine any relations on the base $\mathfrak{K}[[\theta]]=\mathfrak{K} \oplus \mathfrak{K} \theta$, one should compute $\left[d_{1}, d_{1}\right]$, but this is automatically zero since $\theta^{2}=0$. Thus, there are no relations other than the relation $\theta^{2}=0$, which is always true for an odd parameter, and the base of the miniversal deformation is just $A=\mathfrak{K}[\theta]$.

4. Deformations of a $1 \mid 0$ dimensional space. Let $W=\langle e\rangle$ be a one dimensional even vector space. Then $S^{k}(W)=\left\langle e^{k}\right\rangle$, and $L_{k}=\left\langle\varphi_{k}\right\rangle$, where $\varphi_{k}\left(e^{l}\right)=k ! \delta_{k}^{l} e$. Now $\left[\varphi_{k}, \varphi_{l}\right]$ is the element of $L_{k+l-1}$, which we compute in detail, as an illustration of how to perform the computation of brackets. Let $n=k+l-1$. Then

$$
\begin{aligned}
{\left[\varphi_{k}, \varphi_{l}\right]\left(e^{n}\right) } & =\sum_{\sigma \in \operatorname{Sh}(l, n-l)} k ! l ! \varphi_{k}\left(\varphi_{l}\left(e^{l}\right), e^{k-1}\right)-(-1)^{\varphi_{k} \varphi_{l}} \sum_{\sigma \in \operatorname{Sh}(k, l-k)} l ! k ! \varphi_{k}\left(\varphi_{k}\left(e^{k}\right), e^{l-1}\right) \\
& =\left(k ! l !\left(\begin{array}{c}
n \\
l
\end{array}\right)-k ! l !\left(\begin{array}{l}
n \\
k
\end{array}\right)\right) e=(k-l) n ! e=(k-l) \varphi_{n}\left(e^{n}\right) .
\end{aligned}
$$

Since all the maps $\varphi_{k}$ are even, the only codifferential on $W$ is $d=0$. This time, the infinitesimal deformation $d_{1}=\varphi_{k} \theta^{k}$ has an infinite number of terms, and they are not coboundaries. There are some nontrivial relations on the base, arising from the self bracket 
of $d_{1}$. We have

$$
\frac{1}{2}\left[d_{1}, d_{1}\right]=\sum_{n=1}^{\infty} \sum_{k+l=n+1}\left[\varphi_{k} \theta^{k}, \varphi_{l} \theta^{l}\right]=\sum_{n=1}^{\infty} \sum_{k+l=n+1}(k-l) \theta^{k} \theta^{l} \varphi_{n},
$$

from which we obtain an infinite set of relations of the form $\sum_{k+l=n+1}(k-l) \theta^{k} \theta^{l}=0$, for $n=1 \ldots$ Let us examine the first few terms. For $n=1$, the relation is zero. For $n=2$, we obtain $\theta^{2} \theta^{1}-\theta^{1} \theta^{2}=0$, i.e. $\theta^{1} \theta^{2}=0$. For $n=3$, the relation reduces to $\theta^{1} \theta^{3}=0$. For $n=4$, we obtain $6 \theta^{1} \theta^{4}+10 \theta^{2} \theta^{3}=0$. If we let $R=\left\langle\sum_{k+l=n+1}(k-l) \theta^{k} \theta^{l}=0\right\rangle$ be the ideal generated by these relations in $\mathfrak{K}\left[\left[\theta^{1} \ldots\right]\right]$, then the base of the miniversal deformation is just $\mathcal{A}=\mathfrak{K}\left[\left[\theta^{1} \ldots\right]\right] / R$. The miniversal deformation coincides with the universal infinitesimal deformation, except that the base $\mathcal{A}$ of the miniversal deformation is not infinitesimal, but is a somewhat complicated infinite dimensional graded commutative algebra.

5. Deformations of a $2 \mid 0$ dimensional space. Let $W=\left\langle e_{1}, e_{2}\right\rangle$ be a completely even dimensional space. Then $S^{k}(W)=\left\langle e_{1}^{k}, e_{1}^{k-1} e_{2}, \cdots, e_{2}^{k}\right\rangle$ has dimension $k+1$, so that $L_{k}$ has dimension $2(k+1)$. It will simplify the notation if we introduce multi-indices of nonnegative integers $I=\left(i_{1}, i_{2}\right)$. Define $|I|=i_{1}+i_{2}, I !=i_{1} ! i_{2} !, \delta_{J}^{I}=\delta_{j_{1}}^{i_{1}} \delta_{j_{2}}^{i_{2}}$ and $e^{I}=e_{1}^{i_{1}} e_{2}^{i_{2}}$. Then $L_{n}=\left\langle\varphi_{I, i}:|I|=n, i \in\{1,2\}\right\rangle$, where

$$
\varphi_{I, i}\left(e^{J}\right)=I ! \delta_{I}^{J} e_{i}
$$

Note that because $W$ is totally even, the maps $\varphi_{I, i}$ are all even, so that $d=0$ is the only $L_{\infty}$ structure on $W$ and

$$
d_{1}=\varphi_{I, i} \theta^{I, i}
$$

is the universal infinitesimal deformation as well as a miniversal deformation, but in the latter case, we need to compute the relations on the base. To that end, let us compute $\left[\varphi_{I, k}, \varphi_{J, l}\right]$, which lies in $L_{|I|+|J|-1}$. Define $I+J=\left(i_{1}+j_{1}, i_{2}+j_{2}\right)$ and

$$
I-k= \begin{cases}\left(i_{1}-1, i_{2}\right) & \text { if } k=1 \text { and } i_{1}>0 \\ \left(i_{1}, i_{2}-1\right) & \text { if } k=2 \text { and } i_{2}>0\end{cases}
$$

Then

$$
\left[\varphi_{I, k}, \varphi_{J, l}\right]=i_{l} \varphi_{J+(I-l), k}-j_{k} \varphi_{I+(J-k), l},
$$

where terms such that $I-l$ or $J-k$ are not defined are omitted. From this we determine that the relations are given by

$$
\sum_{J+(I-j)=M} i_{j} \theta^{I, m} \theta^{J, j}=0,
$$

for each multi-index $M$ and $m \in\{1,2\}$. This follows from the requirement that $\left[d_{1}, d_{1}\right]=$ 0 . Computing this bracket, we obtain,

$$
\begin{aligned}
\frac{1}{2}\left[d_{1}, d_{1}\right] & =\left(i_{l} \varphi_{J+(I-l), k}-j_{k} \varphi_{I+(J-k), l}\right) \theta^{I, k} \theta^{J, l} \\
& =\sum_{\substack{J+(I-l)=M \\
k=m}} i_{l} \varphi_{M, m} \theta^{I, m} \theta^{J, j}-\sum_{\substack{I+(J-l)=M \\
l=m}} j_{k} \varphi_{M, m} \theta^{I, k} \theta^{J, m} .
\end{aligned}
$$


The second term is the same as the first after interchanging the dummy indices and using the fact that the $\theta$ 's are odd.

6. Deformations of a $0 \mid 2$ dimensional space. Let $W=\left\langle f_{1}, f_{2}\right\rangle$ be a completely odd two dimensional space. Then $S^{2}(W)=\left\langle f_{1} f_{2}\right\rangle$, and $S(W)=W+S^{2}(W)$. We have $L_{1}=\left\langle\varphi_{j}^{i}\right\rangle$, where $\varphi_{j}^{i}\left(f_{k}\right)=\delta_{k}^{i} f_{j}$, and $L_{2}=\left\langle\psi_{i}\right\rangle$, where $\psi_{i}\left(f_{1} f_{2}\right)=f_{i}$. Note that $L_{1}$ consists of even elements, while $L_{2}$ is odd. This time, in addition to the trivial $L_{\infty}$ structure $d=0$, any element of $L_{2}$ also determines an $L_{\infty}$ algebra structure. All of these structures are actually Lie algebra structures, with $d=0$ giving the abelian Lie algebra structure, while the nonzero elements of $L_{2}$ give rise to equivalent structures, so we need only consider the case $d=\psi_{1}$.

As an aid to the construction, we first compute the brackets of all the basis elements. First,

$$
\left[\varphi_{j}^{i}, \varphi_{l}^{k}\right]\left(f_{m}\right)=\varphi_{j}^{i}\left(\varphi_{l}^{k}\left(f_{m}\right)\right)-\varphi_{l}^{k}\left(\varphi_{j}^{i}\left(f_{m}\right)\right)=\delta_{l}^{i} \delta_{m}^{k} f_{j}-\delta_{j}^{k} \delta_{m}^{i} f_{l}
$$

while

$$
\begin{aligned}
{\left[\varphi_{j}^{i}, \psi_{k}\right]\left(f_{1} f_{2}\right) } & =\varphi_{j}^{i}\left(\psi_{k}\left(f_{1} f_{2}\right)\right)-\psi_{k}\left(\varphi_{j}^{i}\left(f_{1}\right) f_{2}\right)+\psi_{k}\left(\varphi_{j}^{i}\left(f_{2}\right) f_{1}\right) \\
& =\varphi_{j}^{i}\left(f_{k}\right)-\psi_{k}\left(\delta_{1}^{i} f_{j} f_{2}\right)+\psi_{k}\left(\delta_{2}^{i} f_{j} f_{1}\right)=\delta_{k}^{i} f_{j}-\delta_{1}^{i} \delta_{j}^{1} f_{k}-\delta_{2}^{i} \delta_{j}^{2} f_{k},
\end{aligned}
$$

so that the brackets are given by

$$
\begin{aligned}
{\left[\varphi_{j}^{i}, \varphi_{l}^{k}\right] } & =\delta_{l}^{i} \varphi_{j}^{k}-\delta_{j}^{k} \varphi_{l}^{i} \\
{\left[\varphi_{j}^{i}, \psi_{k}\right] } & =\delta_{k}^{i} \psi_{j}-\delta_{j}^{i} \psi_{k} \\
{\left[\psi_{i}, \psi_{j}\right] } & =0 .
\end{aligned}
$$

We give a more detailed table of the brackets below, which is useful in determining the cocycles and coboundaries.

$$
\begin{aligned}
& {\left[\varphi_{1}^{1}, \varphi_{1}^{1}\right]=0} \\
& {\left[\varphi_{1}^{1}, \varphi_{2}^{1}\right]=-\varphi_{2}^{1}} \\
& {\left[\varphi_{1}^{1}, \varphi_{1}^{2}\right]=\varphi_{1}^{2} \quad\left[\varphi_{2}^{1}, \varphi_{1}^{2}\right]=\varphi_{2}^{2}-\varphi_{1}^{1}} \\
& {\left[\varphi_{1}^{1}, \varphi_{2}^{2}\right]=0 \quad\left[\varphi_{2}^{1}, \varphi_{2}^{2}\right]=-\varphi_{2}^{1} \quad\left[\varphi_{1}^{2}, \varphi_{2}^{2}\right]=\varphi_{1}^{2}} \\
& {\left[\varphi_{1}^{1}, \psi_{1}\right]=0 \quad\left[\varphi_{2}^{1}, \psi_{1}\right]=\psi_{2} \quad\left[\varphi_{1}^{2}, \psi_{1}\right]=0 \quad\left[\varphi_{2}^{2}, \psi_{1}\right]=-\psi_{1}} \\
& {\left[\varphi_{1}^{1}, \psi_{2}\right]=-\psi_{2} \quad\left[\varphi_{2}^{1}, \psi_{2}\right]=0 \quad\left[\varphi_{1}^{2}, \psi_{2}\right]=\psi_{1} \quad\left[\varphi_{2}^{2}, \psi_{2}\right]=0}
\end{aligned}
$$

6.1. Case $d=0$. For the trivial codifferential $d=0$, every cochain is a cocycle, and therefore, the universal infinitesimal deformation of $d$ is

$$
d_{1}=\varphi_{j}^{i} \theta_{i}^{j}+\psi_{k} t^{k}
$$

where as usual $\theta_{i}^{j}$ are odd parameters and $t_{k}$ are even parameters. Computing the bracket of this derivation with itself, one obtains

$$
\frac{1}{2}\left[d_{1}, d_{1}\right]=-\left(\delta_{k}^{i} \psi_{j}+\delta_{j}^{i} \psi_{k}\right) \theta_{i}^{j} t^{k}+\left(\delta_{n}^{i} \varphi_{j}^{m}-\delta_{j}^{n} \varphi_{m}^{i}\right) \theta_{i}^{j} \theta_{n}^{m} .
$$

Since this bracket must vanish for $d_{1}$ to be a codifferential, we obtain some relations on the parameters. These are more easily seen by examining the detailed table, and looking for which terms contribute to which output cochains. For example, the cochain $\psi_{1}$ only 
arises from the brackets $\left[\varphi_{2}^{2}, \psi_{1}\right]$ and $\left[\varphi_{1}^{2}, \psi_{2}\right]$, and therefore, $\theta_{2}^{2} t^{1}-\theta_{1}^{2} t^{2}=0$. The complete set of relations is given by

$$
R=\left\{\theta_{1}^{1} \theta_{1}^{2} 1+\theta_{1}^{2} \theta_{2}^{2}, \theta_{1}^{1} \theta_{2}^{1}+\theta_{2}^{1} \theta_{2}^{2}, \theta_{2}^{1} \theta_{1}^{2}, \theta_{1}^{1} t^{2}-\theta_{1}^{2} t^{1}, \theta_{2}^{2} t^{1}-\theta_{1}^{2} t^{2}\right\},
$$

and thus the base of the miniversal deformation is $\mathcal{A}=\mathfrak{K}\left[\left[\theta_{j}^{i}, t_{k}\right]\right] / R$.

6.2. Case $d=\psi_{1}$. This second case is the first nontrivial codifferential that we have encountered. From the table of brackets, we see that $\left\{\varphi_{1}^{1}, \varphi_{1}^{2}, \psi_{1}, \psi_{2}\right\}$ is a basis of the space of cocycles, with $\left\{\psi_{1}, \psi_{2}\right\}$ being a basis of the coboundaries. Thus $\left\{\varphi_{1}^{1}, \varphi_{1}^{2}\right\}$ projects to a basis of the cohomology of $d$, which is two dimensional. The universal infinitesimal deformation of $d$ is given by $d_{1}=\psi_{1}+\varphi_{1}^{1} \theta_{1}^{1}+\varphi_{1}^{2} \theta_{2}^{1}$. Since $\left[d_{1}, d_{1}\right]=2 \varphi_{1}^{2} \theta_{1}^{1} \theta_{2}^{1}$, we obtain only one relation, $R=\left\{\theta_{1}^{1} \theta_{2}^{1}\right\}$, so that the infinitesimal deformation is miniversal, and $\mathcal{A}=\mathfrak{K}\left[\theta_{1}^{1}, \theta_{2}^{1}\right] / R=\mathfrak{K}+\mathfrak{K} \theta_{1}^{1}+\mathfrak{K} \theta_{2}^{1}$ is the base of the versal deformation. This completes the description of the miniversal deformation; its formula coincides with that of the universal infinitesimal deformation. We would like to explore this example a bit further.

We will consider a different infinitesimal deformation, which we will extend to a formal deformation. Since the infinitesimal deformation we have already given is universal, we start by adding some coboundary terms to our original deformation. We begin with

$$
d_{1}^{\prime}=\psi_{1}+\varphi_{1}^{1} \theta_{1}^{1}+\varphi_{1}^{2} \theta_{2}^{1}+\psi_{1} t^{1}+\psi_{2} t^{2},
$$

and proceed to construct a formal deformation out of this infinitesimal deformation of $d$. Since we added merely a coboundary term, this deformation is equivalent in some sense to the original one, but note that the base of this infinitesimal deformation is different, since there are more parameters.

We first carry out the construction of a formal deformation from the above infinitesimal one, and then show how to construct the homomorphism from the base of the miniversal deformation to the base of the formal deformation we are about to construct. Then we will explicitly determine the equivalence between the push out of $d$ given by the homomorphism of the bases and the formal deformation $d_{\infty}^{\prime}$ which is obtained by extending the infinitesimal $d_{1}^{\prime}$ to a formal one. The authors found this construction intriguing, because the obvious homomorphism between the bases does not turn out to be the correct one. In addition, this example illustrates how to extend a deformation to higher order by adding higher order terms to eliminate coboundary terms arising in the computation of the self-bracket of the codifferential. Up to now, all of the terms arising in these computations have been coboundary free, so that they generate only relations, which means that the associated formal deformations are really infinitesimal ones and thus don't provide a good illustration of the theory of formal deformations.

Let us first compute the bracket $\left[d_{1}^{\prime}, d_{1}^{\prime}\right]$ of the infinitesimal deformation. We have

$$
\begin{aligned}
\frac{1}{2}\left[d_{1}^{\prime}, d_{1}^{\prime}\right] & =\left[\varphi_{1}^{1}, \varphi_{1}^{2}\right] \theta_{1}^{1} \theta_{2}^{1}-\left[\varphi_{1}^{1}, \psi_{2}\right] \theta_{1}^{1} t^{2}-\left[\varphi_{1}^{2}, \psi_{2}\right] \theta_{2}^{1} t^{2}=\varphi_{1}^{2} \theta_{1}^{1} \theta_{2}^{1}+\psi_{2} \theta_{1}^{1} t^{2}-\psi_{1} \theta_{2}^{1} t^{2} \\
& =\varphi_{1}^{2} \theta_{1}^{1} \theta_{2}^{1}-D\left(\varphi_{2}^{1}\right) \theta_{1}^{1} t^{2}-D\left(\varphi_{2}^{2}\right) \theta_{2}^{1} t^{2} .
\end{aligned}
$$

The second order deformation is given by extending $d_{1}^{\prime}$ to

$$
d_{2}^{\prime}=\psi_{1}+\varphi_{1}^{1} \theta_{1}^{1}+\varphi_{1}^{2} \theta_{2}^{1}+\psi_{1} t^{1}+\psi_{2} t^{2}+\varphi_{2}^{1} \theta_{1}^{1} t^{2}+\varphi_{2}^{2} \theta_{2}^{1} t^{2} .
$$


There is one necessary relation on the parameters: $\theta_{1}^{1} \theta_{2}^{1}=0$. Note that formally speaking, this relation should be interpreted as being only up to order 2 , so that in the formal deformation, the relation that corresponds to this one could, a priori, pick up some higher order terms.

One could continue in the same manner to compute a third order deformation, but note that if the expression above is bracketed with itself, since $\psi_{2}$ kills $\varphi_{2}^{1}$ and $\varphi_{2}^{2}$, and $\psi_{1}$ takes $\varphi_{2}^{1}$ to $\psi_{2}$ and $\varphi_{2}^{2}$ to $\psi_{1}$, we will end up only adding more terms involving $\varphi_{2}^{1}$ and $\varphi_{2}^{2}$. Examining the terms in the bracket which involve $\varphi_{1}^{1}$ and $\varphi_{1}^{2}$ one determines that the same relation $\theta_{1}^{1} \theta_{2}^{1}=0$ holds (up to third order) and no additional relations are necessary. Thus it is reasonable to expect that one can write the formal deformation in the form

$$
d_{\infty}^{\prime}=\psi_{1}\left(1+t^{1}\right)+\varphi_{1}^{1} \theta_{1}^{1}+\varphi_{1}^{2} \theta_{2}^{1}+\psi_{2} t^{2}+\varphi_{2}^{1} \theta_{1}^{1} t^{2} c_{1}+\varphi_{2}^{2} \theta_{2}^{1} t^{2} c_{2},
$$

and solve for the constants $c_{1}, c_{2}$ and the relations on the parameters necessary so that $\left[d_{\infty}^{\prime}, d_{\infty}^{\prime}\right]=0$. The solution is given by $c_{1}=c_{2}=\frac{1}{1+t^{1}}$, which should be considered as a formal power series in $t^{1}$. The base of this formal deformation is $\mathcal{A}^{\prime}=\mathfrak{K}\left[\left[\theta_{2}^{1}, \theta_{1}^{1}, t^{1}, t^{2}\right]\right] /\left(\theta_{1}^{1} \theta_{2}^{1}\right)$.

Since the deformation $d_{1}$ is miniversal, there must be some morphism $\lambda: \mathcal{A} \rightarrow \mathcal{A}^{\prime}$, such that $\lambda_{*}\left(d_{1}\right) \cong d_{\infty}^{\prime}$. This means that there is some automorphism $\tilde{g}$ of $S(W)$ such that $d_{\infty}^{\prime}=\tilde{g}^{-1} \lambda_{*}\left(d_{1}\right) \tilde{g}$. Suppose that $\tilde{g}$ is determined by an isomorphism $g: W \otimes \mathcal{A}^{\prime} \rightarrow W \otimes \mathcal{A}^{\prime}$ of the form $g=I+u_{1} \varphi_{2}^{1}+u_{2} \varphi_{2}^{2}$, for some constants $u_{1}$ and $u_{2}$. First we show that this is indeed an invertible map, by constructing its inverse $g^{-1}=I+v_{1} \varphi_{2}^{1}+v_{2} \varphi_{2}^{2}$. It is easily checked that $v_{1}=\frac{-u_{1}}{1+u_{2}}, v_{2}=\frac{-u_{2}}{1+u_{2}}$ determine the inverse map. Next, note that $\tilde{g}=g+g \odot g$, where $g \odot g: S^{2}(W) \rightarrow S^{2}(W)$ is the map given by $(g \odot g)(a b)=g(a) g(b)$. In our case, we compute

$$
(g \odot g)\left(f_{1} f_{2}\right)=g\left(f_{1}\right) g\left(f_{2}\right)=\left(f_{1}+u_{1} f_{2}\right)\left(f_{2}+u_{2} f_{2}\right)=\left(1+u_{2}\right)\left(f_{1} f_{2}\right),
$$

so that $g \odot g=\left(1+u_{2}\right) I \odot I$. Let us denote $\lambda\left(\theta_{1}^{1}\right)$ by $\tilde{\theta}_{1}^{1}$ and $\lambda\left(\theta_{2}^{1}\right)$ by $\tilde{\theta}_{2}^{1}$, so that $\lambda_{*}\left(d_{1}\right)=\psi_{1}+\varphi_{1}^{1} \tilde{\theta}_{1}^{1}+\varphi_{1}^{2} \tilde{\theta}_{2}^{1}$. Then

$$
\lambda_{*}\left(d_{1}\right) \tilde{g}=\left(1+u_{2}\right) \psi_{1}+\varphi_{1}^{1}\left(\tilde{\theta}_{1}^{1}+u_{1} \tilde{\theta}_{2}^{1}\right)+\varphi_{1}^{2}\left(1+u_{2}\right) \tilde{\theta}_{2}^{1} .
$$

Finally, we compute that

$$
\begin{aligned}
g^{-1} \lambda_{*}\left(d_{1}\right) \tilde{g}= & \left(1+u_{2}\right) \psi_{1}+\varphi_{1}^{1}\left(\tilde{\theta}_{1}^{1}+u_{1} \tilde{\theta}_{2}^{1}\right)+\varphi_{1}^{2}\left(1+u_{2}\right) \tilde{\theta}_{2}^{1} \\
& +v_{1}\left(1+u_{2}\right) \psi_{2}+\varphi_{2}^{1} v_{1}\left(\tilde{\theta}_{1}^{1}+u_{1} \tilde{\theta}_{2}^{1}\right)+\varphi_{2}^{2} v_{1}\left(1+u_{2}\right) \tilde{\theta}_{2}^{1}
\end{aligned}
$$

To get the correct image we must have $\theta_{1}^{1}=\tilde{\theta}_{1}^{1}+u_{1} \tilde{\theta}_{2}^{1}, \theta_{2}^{1}=\left(1+u_{2}\right) \tilde{\theta}_{2}^{1}, u_{2}=t^{1}$ and $u_{1}=-t^{2}$ (which finally justifies our tacit assumption that $1+u_{2}$ is invertible in $\mathcal{A}^{\prime}$ ). Thus the map $\lambda$ is given by

$$
\lambda\left(\theta_{2}^{1}\right)=\frac{1}{1+t^{1}} \theta_{2}^{1}, \quad \lambda\left(\theta_{1}^{1}\right)=\theta_{1}^{1}+\frac{t^{2}}{1+t^{1}} \theta_{2}^{1} .
$$

Note that this map is not even polynomial!

We computed the morphism $\lambda$ from $\mathcal{A}$ to the formal algebra $\mathcal{A}^{\prime}$, but the same ideas can be used to compute the morphism to the algebra associated with the $n$-th order deformation. In particular, one can compute the infinitesimal morphism. But all of these morphisms are just the $\bmod \mathfrak{m}^{k}$ reductions of the morphism $\lambda$ given above. 
Even though our claim that any nonzero linear combination of the cochains $\psi_{1}$ and $\psi_{2}$ is equivalent as an $L_{\infty}$ algebra structure to $d=\psi_{1}$, is just a classical fact about Lie algebras, it can be demonstrated easily using our methodology involving a coalgebra morphism $g=I+u_{1} \varphi_{2}^{1}+u_{2} \varphi_{2}^{2}$. If we compute $g^{-1} \circ d \circ \tilde{g}$ as before, we obtain simply $g^{*}(d)=\left(1+u_{2}\right) \psi_{1}-u_{2} \psi_{2}$, which means that any combination for which the coefficient of $\psi_{1}$ is nonzero can be obtained (recall that $1+u_{2}$ needs to be invertible). It is also easy to see that if we take $g=\varphi_{2}^{1}+\varphi_{1}^{2}$, then $g^{-1}=g, \tilde{g}=g+I \odot I$, and $g^{*}\left(\psi_{1}\right)=\psi_{2}$.

7. Deformations of a $1 \mid 1$ dimensional space. If $W=\langle e, f\rangle$ is a $1 \mid 1$ dimensional space, then $S^{k}(W)=\left\langle e^{k}, e^{k-1} f\right\rangle$ is a $1 \mid 1$ dimensional space as well, and $L_{k}=$ $\left\langle\varphi_{e}^{k}, \varphi_{f}^{k}, \psi_{e}^{k} \psi_{f}^{k},\right\rangle$ is a $2 \mid 2$ dimensional space, where

$$
\begin{array}{lr}
\varphi_{e}^{k}\left(e^{k}\right)=k ! e & \psi_{e}^{k}\left(e^{k-1} f\right)=(k-1) ! e \\
\varphi_{f}^{k}\left(e^{k-1} f\right)=(k-1) ! f & \psi_{f}^{k}\left(e^{k}\right)=k ! f .
\end{array}
$$

The bracket structure is given by the table below.

$$
\begin{array}{lll}
{\left[\varphi_{e}^{m}, \varphi_{e}^{n}\right]=(m-n) \varphi_{e}^{m+n-1}} & {\left[\varphi_{e}^{m}, \varphi_{f}^{n}\right]=(1-n) \varphi_{f}^{m+n-1}} \\
{\left[\varphi_{f}^{m}, \varphi_{f}^{n}\right]=0} & & \\
{\left[\varphi_{e}^{m}, \psi_{e}^{n}\right]=(m-n+1) \psi_{e}^{m+n-1}} & {\left[\varphi_{f}^{m}, \psi_{e}^{n}\right]=-\psi_{e}^{m+n-1}} \\
{\left[\varphi_{e}^{m}, \psi_{f}^{n}\right]=-n \psi_{f}^{m+n-1}} & {\left[\varphi_{f}^{m}, \psi_{f}^{n}\right]=\psi_{f}^{m+n-1}} \\
{\left[\psi_{e}^{m}, \psi_{f}^{n}\right]=\varphi_{e}^{m+n-1}+n \varphi_{f}^{m+n-1}} & \\
{\left[\psi_{e}^{n}, \psi_{e}^{n}\right]=0} & {\left[\psi_{f}^{n}, \psi_{f}^{n}\right]=0}
\end{array}
$$

Let us first take care of the simple case when $d=0$. Since every cochain is a cocycle and there are no coboundaries, the universal infinitesimal extension is given by

$$
d_{1}=\varphi_{e}^{n} \theta_{n}^{e}+\varphi_{f}^{n} \theta_{n}^{f}+\psi_{e}^{n} t_{n}^{e}+\psi_{f}^{n} t_{n}^{f} .
$$

This is also the formula for a miniversal deformation, but then the following relations on the base are necessary.

$$
\begin{array}{ll}
\sum_{k+l=n+1}(k-l) \theta_{k}^{e} \theta_{l}^{e}+t_{k}^{e} t_{l}^{f}=0 & \sum_{k+l=n+1}-(k-l+1) \theta_{k}^{e} t_{l}^{e}+\theta_{k}^{f} t_{l}^{e}=0 \\
\sum_{k+1=n+1}-(l-1) \theta_{k}^{e} \theta_{l}^{f}+l t_{k}^{e} t_{l}^{f}=0 & \sum_{k+l=n+1} \theta_{k}^{e} t_{l}^{f}-\theta_{k}^{f} t_{l}^{f}=0
\end{array}
$$

Having disposed of the trivial codifferential, we now consider the general case $d=$ $r_{n} \psi_{e}^{n}+s_{n} \psi_{f}^{n}$ for some constants $r_{n}, s_{n} \in \mathfrak{K}$. Then a computation of $[d, d]$ yields

$$
\frac{1}{2}[d, d]=\sum_{n=1}^{\infty} \sum_{k+l=n+1} r_{k} s_{l}\left(\varphi_{e}^{n}+l \varphi_{f}^{n}\right),
$$

from which it follows that $\sum_{k+l=n+1} r_{k} s_{l}=0$ for all $n$. Let $M$ be the least integer such that not both $r_{M}$ and $s_{M}$ vanish. If $r_{M} \neq 0$, then it is easy to see that this forces $s_{l}=0$ for all $l$. A similar result holds if $s_{M} \neq 0$. Thus the only possibilities for nontrivial codifferentials are of the form $d=r_{k} \psi_{e}^{k}$ and $d=s_{k} \psi_{f}^{k}$.

Let us consider the case $d=r_{k} \psi_{e}^{k}$ first. Let $L$ be the least coefficient such that $r_{L} \neq 0$, and for simplicity, assume that $r_{L}=1$. Let $d^{L}=\psi_{L}^{k}$. Note that $\psi_{e}^{L+k}=\left[d^{L}, \varphi_{f}^{k+1}\right]$, so that $d=d^{L}+\left[d^{L}, \sum_{k=1}^{\infty} r_{k+L} \psi_{f}^{k+1}\right]$. This resembles the case of an infinitesimal deformation, 
suggesting that $d$ is equivalent to $d^{L}$. Let $\tilde{\varphi}_{f}^{k}$ represent the coderivation associated to $\varphi_{f}^{k}$ (recall that $\varphi_{f}^{k} \in \operatorname{Hom}(S(W), W)$ ). Then the bracket is given by $\left[d^{L}, \varphi_{f}^{k}\right]=d^{L} \circ \tilde{\varphi}_{f}^{k}$. This is because $d^{L}$ vanishes on terms that do not contain an $f$, but outputs no $f$, while $\varphi_{f}^{k}$ requires an input of an $f$. So in the decomposition of the bracket, only one of the terms survives.

Let $\tilde{\lambda}=\sum_{k=1}^{\infty} r_{k+L} \tilde{\varphi}_{f}^{k+1}$. Then $\tilde{\lambda}$ is a coderivation of $S(W)$, so that

$$
\Delta \circ \tilde{\lambda}=(\tilde{\lambda} \otimes I+I \otimes \tilde{\lambda}) \circ \Delta .
$$

Let $\tilde{g}=I+\tilde{\lambda}$. Then we claim that $\tilde{g}$ is an automorphism of $S(W)$. For that to be true, the identity that needs to be satisfied is

$$
\Delta \circ \tilde{\lambda}=(\tilde{\lambda} \otimes I+I \otimes \tilde{\lambda}+\tilde{\lambda} \otimes \tilde{\lambda}) \circ \Delta
$$

but this holds since $(\tilde{\lambda} \otimes \tilde{\lambda}) \circ \Delta=0$. To see this fact, note that $\tilde{\lambda}$ vanishes on an element in $S(W)$ not containing an $f$, while only one of the two terms output by $\Delta$ could contain an $f$, since $f^{2}=0$. If $\tilde{\lambda}$ was an infinitesimal derivation, then $\tilde{g}^{-1}$ would simply be $I-\tilde{\lambda}$. This is not true here. Nevertheless, we can compute $\tilde{g}^{-1}$ exactly. For simplicity, first consider the case $L=1$ and $\tilde{\lambda}=\tilde{\varphi}_{f}^{2}$. Now,

$$
\tilde{\lambda} \varphi_{f}^{k}\left(e^{n} f\right)=(k-1) !\left(\begin{array}{c}
n \\
k-1
\end{array}\right) e^{n-k} f
$$

so $\tilde{g}\left(e^{n} f\right)=e^{n} f+\left(\begin{array}{c}n \\ 1\end{array}\right) e^{n-1} f$. It follows that

$$
\varphi_{f}^{k}\left(e^{n} f+\left(\begin{array}{c}
n \\
1
\end{array}\right) e^{n-1}\right)=(k-1) !\left(\begin{array}{c}
n \\
k-1
\end{array}\right) e^{n-k} f+(k-1) !\left(\begin{array}{c}
n-1 \\
k-1
\end{array}\right)\left(\begin{array}{c}
n \\
1
\end{array}\right) e^{n-k-1} f .
$$

Since $k !\left(\begin{array}{l}n \\ k\end{array}\right)=(k-1) !\left(\begin{array}{l}n-1 \\ k-1\end{array}\right)\left(\begin{array}{l}n \\ 1\end{array}\right)$, it is easily seen that $\tilde{g}^{-1}=I-\sum_{k=2}^{\infty}(-1)^{i} \varphi_{f}^{k}$. For arbitrary $L$ and $\tilde{\lambda}$, we can express $\tilde{g}^{-1}=I+\sum_{k=2}^{\infty} c_{k} \varphi_{f}^{k}$, for some constants $c_{k}$.

From the consideration given above on how $\varphi_{f}^{k}$ interacts $d^{L}$, we see that $d=\tilde{g}^{-1} \circ d^{L} \circ \tilde{g}$; in other words, $d$ and $d^{L}$ determine equivalent $L_{\infty}$ structures.

A similar argument applies to the case of $s_{k} \psi_{f}^{k}$, except that in this case, one sees that $\psi_{f}^{k} \circ \tilde{\varphi}_{f}^{l}=0$, because $\varphi_{f}^{l}$ always inputs an $f$ and outputs one, while $\psi_{f}^{k}$ inputs no $f$ and outputs one, so the composition is zero. Thus the bracket should be computed in the form $d=\tilde{g} \circ \psi_{f}^{L} \circ \tilde{g}^{-1}$ for an appropriate choice of $\tilde{g}$.

The $L_{\infty}$ structures determined by $\psi_{e}^{k}$ and $\psi_{f}^{l}$ can never be equivalent. To see this, note that if we extend these maps to coderivations, then we have

$$
\begin{array}{ll}
\tilde{\psi}_{f}^{k}\left(e^{n}\right)=\left(\begin{array}{l}
n \\
k
\end{array}\right) e^{n-k} f & \tilde{\psi}_{f}^{k}\left(e^{n} f\right)=0 \\
\tilde{\psi}_{e}^{k}\left(e^{n} f\right)=\left(\begin{array}{c}
n \\
k-1
\end{array}\right) e^{n-k+2} & \tilde{\psi}_{e}^{k}\left(e^{n}\right)=0
\end{array}
$$

Suppose that $\tilde{\psi}_{f}^{l}=\tilde{g}^{-1} \circ \tilde{\psi}_{e}^{k} \circ \tilde{g}$. Then $\tilde{g} \circ \tilde{\psi}_{f}^{l}=\tilde{\psi}_{e}^{k} \circ \tilde{g}$. Since $\tilde{g} \circ \tilde{\psi}_{f}^{l}\left(e^{n} f\right)=0$, it follows that $\tilde{g}\left(e^{n} f\right)$ must lie in the kernel of $\tilde{\psi}_{e}^{k}$. But this implies that $\tilde{g}\left(e^{n} f\right)$ would be even. This is impossible since an automorphism is an even map, and $e^{n} f$ is odd. Thus no such equivalence is possible.

Next, we will compute the cohomology $H\left(d_{k}\right)$ for $d_{k}=\varphi_{e}^{k}$, and show that it has dimension $2 L-2$. From this, it follows immediately that if $k \neq l$, then $d_{k}$ is not equivalent to $d_{l}$. A similar result holds for $d_{k}=\varphi_{f}^{k}$. Thus there are two infinite families of 
nonequivalent $L_{\infty}$ structures on $W$. It is very interesting that such a simple space gives rise to so many distinct $L_{\infty}$ structures.

7.1. Case $d^{L}=\psi_{e}^{L}$. First, note that $D_{L}\left(\psi_{e}^{k}\right)=0$, so $\psi_{e}^{k}$ is a cocycle for all $k$. No element of order less than $L$ can be a coboundary, because the bracket of anything with $d^{L}$ has order at least $L$. Since $D_{L}\left(\varphi_{f}^{k}\right)=\psi_{f}^{m+L-1}, \psi_{e}^{k}$ is a coboundary if $k \geq L$. Since $\psi_{f}^{k}$ is never a cocycle, it is clear that the odd cocycles are spanned by $\psi_{e}^{k}$, and the dimension of the odd part of the cohomology of $d^{L}$ is $L-1$.

Let $h^{k}=\varphi_{e}^{k}+(k-L+1) \varphi_{f}^{k}$. Then $D_{L}\left(h^{k}\right)=0$, and moreover $D_{L}\left(\psi_{f}^{k-L+1}\right)=h^{k}$, when $k \geq L$, so $h^{k}$ is a coboundary precisely when $k \geq L$. Assume that $\varphi=r_{k} \varphi_{e}^{k}+s_{k} \varphi_{f}^{k}$ is an arbitrary even cocycle. Then $\varphi-r_{k} h^{k}=\left(s_{k}-r_{k}(k-L+1)\right) \varphi_{f}^{k}$, so because $D_{L}\left(\varphi_{f}^{k}\right) \neq 0$, it follows that $s_{k}=r_{k}(k-L+1)$ for all $k$, and thus $\varphi=r_{k} h^{k}$. The cocycles $h^{k}$ form a basis of the even cocycles, so the dimension of the even part of the cohomology of $d^{L}$ is also $L-1$. Thus $\operatorname{dim}\left(H\left(d^{L}\right)\right)=2 L-2$.

If we consider $d^{L}=\psi_{f}^{L}$ instead of $\psi_{e}^{L}$, then the odd cocycles would be given by $\psi_{f}^{k}$, with those where $k \geq L$ being coboundaries, while the even cocycles would be given by $h^{k}=\varphi_{e}^{k}+L \varphi_{f}^{k}, D_{L}\left(\psi_{e}^{k-L+1}\right)=h^{k}$ for $k \geq L$. Thus we obtain the same dimension $2 L-2$ for the cohomology determined by $\psi_{f}^{L}$.

Now let us work with the case $d^{L}=\psi_{e}^{L}$ and calculate a miniversal deformation for some small values of $L$. It will prove useful to have a table of some brackets of $h^{k}$ with certain cochains.

$$
\begin{array}{ll}
{\left[h^{k}, h^{l}\right]=(k-l) h^{k+l-1}} & {\left[h^{k}, \psi_{e}^{l}\right]=(L-l) \psi_{e}^{k+l-1}} \\
{\left[h^{k}, \varphi_{f}^{l}\right]=(1-l) \varphi_{f}^{k+l-1}} & {\left[h^{k}, \psi_{f}^{l}\right]=(k-l-L+1) \psi_{f}^{k+l-1}}
\end{array}
$$

7.2. Case $d=\psi_{e}^{1}$. If $L=1$, then since $H\left(d^{1}\right)=0$, the miniversal deformation is just $d^{1}$. This corresponds to the fact that $W$ has a differential equipping it with the structure of a differential graded vector space, and this differential is essentially unique.

7.3. Case $d=\psi_{e}^{2}$. If $L=2$, then the universal infinitesimal deformation of $d^{1}$ is $d_{1}^{1}=$ $\psi_{e}^{2}+h^{1} \theta_{1}+\psi_{e}^{1} t_{1}$, and $\left[d_{1}^{1}, d_{1}^{1}\right]=-\psi_{e}^{1} \theta_{1} t_{1}$. Thus the infinitesimal deformation is miniversal, with the relation $\theta_{1} t_{1}=0$. Note that in this case, $\psi_{e}^{2}$ determines a nontrivial $\mathbb{Z}_{2}$-graded Lie algebra structure on the parity reversion of $W$, and $\psi_{e}^{1}$ corresponds to the fact that the differential on $W$ is a derivation of this Lie algebra structure. Thus the miniversal deformation gives a deformation of the Lie algebra structure into an $L_{\infty}$ algebra by recording the graded derivations of the Lie algebra structure. The part contributed by $h^{1}$ corresponds to a $\mathbb{Z}_{2}$-graded antiderivation of the Lie algebra, which becomes a true derivation only when it is multiplied by an odd parameter.

7.4. Case $d=\psi_{e}^{3}$. For $L=3$ the universal infinitesimal deformation of $d^{3}$ is

$$
d_{1}^{3}=\psi_{e}^{3}+h^{1} \theta_{1}+h^{2} \theta_{2}+\psi_{e}^{1} t_{1}+\psi_{e}^{2} t_{2} .
$$

From

$$
\frac{1}{2}\left[d_{1}^{3}, d_{1}^{3}\right]=-h^{2} \theta_{1} \theta_{2}-2 \psi_{e}^{1} \theta_{1} t_{1}-\psi_{e}^{2}\left(\theta_{1} t_{2}+2 \theta_{2} t_{1}\right)-\psi_{e}^{3} \theta_{2} t_{2}
$$

we obtain the $\bmod \mathfrak{m}^{3}$ relations $R-2=\left\{\theta_{1} \theta_{2}, \theta_{1} t_{1}, \theta_{1} t_{2}+2 \theta_{2} t_{1}\right\}$ corresponding to the coefficients of the $h^{2}, \psi_{e}^{1}$ and $\psi_{e}^{2}$ terms. Since $\psi_{e}^{3}$ is a coboundary, it does not give a 
relation, but means that we need to add a second order term to the codifferential. In this example the miniversal deformation is not given by the same formula as the universal infinitesimal deformation. The second order deformation $d_{2}^{3}$ is given by the formula

$$
d_{2}^{3}=\psi_{e}^{3}+h^{1} \theta_{1}+h^{2} \theta_{2}+\psi_{e}^{1} t_{1}+\psi_{e}^{2} t_{2}+\varphi_{f}^{1} \theta_{2} t_{2} .
$$

Finally, we compute

$$
\frac{1}{2}\left[d_{2}^{3}, d_{2}^{3}\right]=-h^{2} \theta_{1} \theta_{2}-\psi_{e}^{1}\left(2 \theta_{1} t_{1}-\theta_{2} t_{1} t_{2}\right)-\psi_{e}^{2}\left(\theta_{1} t_{2}+2 \theta_{2} t_{1}-\theta_{2} t_{2}^{2}\right) .
$$

This time no coboundary terms arise in the bracket, so that the miniversal deformation is given by $d_{2}^{3}$, subject to the relations

$$
R_{3}=\left\{\theta_{1} \theta_{2}, 2 \theta_{1} t_{1}-\theta_{2} t_{1} t_{2}, \theta_{1} t_{2}+2 \theta_{2} t_{1}-\theta_{2} t_{2}^{2}\right\} .
$$

Notice that the relations come from the $\bmod \mathfrak{m}^{4}$ relations, but that they are modified by picking up third order terms. This point was misstated in [7], where it was declared that any relations discovered at $n$-th order remain relations at all orders. What really happens is that if you reduce the relations in the formal algebra mod $\mathfrak{m}^{n+1}$, then they become the $n$-th order relations.

7.5. Case $d=\psi_{e}^{4}$. For $L=4$ the universal infinitesimal deformation of $d^{4}$ is

$$
d_{1}^{4}=\psi_{e}^{4}+h^{1} \theta_{1}+h^{2} \theta_{2}+h^{3} \theta^{3}+\psi_{e}^{1} t_{1}+\psi_{e}^{2} t_{2}+\psi_{e}^{3} t_{3} .
$$

Then

$$
\begin{aligned}
\frac{1}{2}\left[d_{1}^{4}, d_{1}^{4}\right]= & -h^{2} \theta_{1} \theta_{2}-2 h^{3} \theta_{1} \theta_{3}-h^{4} \theta_{2} \theta_{3}-3 \psi_{e}^{1} \theta_{1} t_{1}-\psi_{e}^{2}\left(2 \theta_{1} t_{2}+3 \theta_{2} t_{1}\right) \\
& -\psi_{e}^{3}\left(\theta_{1} t_{3}+2 \theta_{2} t_{2}+3 \theta_{3} t_{1}\right)-\psi_{e}^{4}\left(\theta_{2} t_{3}+2 \theta_{3} t_{2}\right)-\psi_{e}^{5} \theta_{3} t_{3} .
\end{aligned}
$$

The coefficients of $h^{k}$ and $\psi_{e}^{k}$ for $k=1,2,3$ yield $5 \bmod \mathfrak{m}^{3}$ relations:

$$
R_{2}=\left\{\theta_{1} \theta_{2}, \theta_{1} \theta_{3}, \theta_{1} t_{1}, 2 \theta_{1} t_{2}+3 \theta_{2} t_{1}, \theta_{1} t_{3}+2 \theta_{2} t_{2}+3 \theta_{3} t_{1} \cdot\right\}
$$

There are also 3 coboundary terms, so that after adding terms to cancel them we arrive at the following formula for the second order deformation.

$$
d_{2}^{4}=d_{1}^{4}+\psi_{f}^{1} \theta_{2} \theta_{3}+\varphi_{f}^{1}\left(\theta_{2} t_{3}+2 \theta_{3} t_{2}\right)+\varphi_{f}^{2} \theta_{3} t_{3} .
$$

The bracket relation $\left[\psi_{e}^{k}, \psi_{f}^{l}\right]=h^{k+l-1}+(L-k) \varphi_{f}^{k+l-1}$, which is easy to verify, will be used in the calculations below.

$$
\begin{aligned}
\frac{1}{2}\left[d_{2}^{4}, d_{2}^{4}\right]= & h^{1} \theta_{2} \theta_{3} t_{1}+h^{2}\left(-\theta_{1} \theta_{2}+\theta_{2} \theta_{3} t_{2}\right)+h^{3}\left(-2 \theta_{1} \theta_{3}+\theta_{2} \theta_{3} t_{3}\right) \\
& +\psi_{e}^{1}\left(-3 \theta_{1} t_{1}+\theta_{2} t_{1} t_{3}+2 \theta_{3} t_{1} t_{2}\right) \\
& +\psi_{e}^{2}\left(-2 \theta_{1} t_{2}-3 \theta_{2} t_{1}+\theta_{2} t_{2} t_{3}+2 \theta_{3} t_{2}^{2}+\theta_{3} t_{1} t_{3}\right) \\
& +\psi_{e}^{3}\left(-\theta_{1} t_{3}-2 \theta_{2} t_{2}-3 \theta_{3} t_{1}+\theta_{2} t_{3}^{2}+3 \theta_{3} t_{2} t_{3}\right) \\
& +3 \varphi_{f}^{1} \theta_{2} \theta_{3} t_{1}+\varphi_{f}^{2}\left(2 \theta_{2} \theta_{3} t_{2}-\theta_{1} \theta_{3} t_{3}\right)+\psi_{e}^{4} \theta_{3} t_{3}^{2}
\end{aligned}
$$

The $\bmod \mathfrak{m}^{4}$ relations are the coefficients of the $h^{k}$ and $\psi_{e}^{k}$ terms for $k=1,2,3$, so there are six of them. But what about the terms that involve $\varphi_{f}^{1}$ and $\varphi_{f}^{2}$ ? These cochains are not even cocycles, so what are they doing in the expression at all? But if you check carefully, the $\bmod \mathfrak{m}^{4}$ relations show that the coefficients are zero $\left(\bmod \mathfrak{m}^{4}\right)$. Thus these terms do not contribute to the bracket at this level. Finally, there is one coboundary term left, so 
it is necessary to add another term, and we have

$$
d_{3}^{4}=d_{2}^{4}-\varphi_{f}^{1} \theta_{3} t_{3}^{2} .
$$

After computing the boundary, the relations given by the coefficients of $\psi_{e}^{1}, \psi_{e}^{2}$ and $\psi_{e}^{3}$ are modified by adding the fourth order term $-\theta_{3} t_{i} t_{3}^{2}$ to the coefficient of $\psi_{e}^{i}$. The coefficients of the cocycles which are not coboundaries determine six relations on the base which is of the form $\mathcal{A}=\mathfrak{K}\left[\left[\theta_{1}, \theta_{2}, \theta_{3}, t_{1}, t_{2}, t_{3}\right]\right] / R$. The coefficient of $\varphi_{f}^{1}$ is one of the relations, so it is clearly zero. It is also easy to show that the coefficient of $\varphi_{f}^{2}$ is equal to zero $\bmod$ $\mathfrak{m}^{4}$, but since we are claiming that the deformation is miniversal, the coefficient must be exactly equal to zero. Multiplying the coefficient of $\psi_{f}^{3}$ by $\theta_{3}$ yields

$$
\theta_{1} \theta_{3} t_{3}+2 \theta_{2} \theta 3 t_{2}-\theta_{2} \theta_{3} t_{3}^{2}=\theta_{1} \theta_{3} t_{3}+2 \theta_{2} \theta_{3} t_{2}-2 \theta_{1} \theta_{3} t_{3}=2 \theta_{2} \theta_{3} t_{2}-\theta_{1} \theta_{3} t_{3},
$$

where we used the relation $\theta_{2} \theta_{3} t_{3}=2 \theta_{1} \theta_{3}$ in the second step. This is precisely the coefficient of $\varphi_{f}^{2}$, so the coefficient of this term is zero. (Of course, it is really not necessary to show that these coefficients are zero, because it follows from the general theory that this must be the case.) Thus $d^{4}$ gives a miniversal deformation. Note that all terms in the relations have odd parameters in them. These computations illustrate the importance of introducing odd parameters, because otherwise $d_{1}$ would be a miniversal deformation.

It is not obvious how to write down the formula for the miniversal deformation of $d^{L}$ in general, but there are some things which can be easily shown in the general case. First, there will be $2 L-2$ relations, (when $L \geq 4$ ). Secondly, by studying the examples so far, it can be seen that the $(L-1)$-th order deformation is miniversal. Thus only a finite number of computations is necessary in order to compute the miniversal deformation.

8. Conclusions. In the examples we have studied, we have given explicit constructions of miniversal deformations. Previous works have only computed the base of a miniversal deformation. The main complication in these constructions is the requirement of exact knowledge of the bracket structure on the space of cochains. Since most of the examples in the literature where cohomology of Lie algebras is studied do not contain this information, the computation of versal deformations of these structures will require more information than is currently easily available. We have just touched on the beginning of the subject.

In a future work, the authors plan to investigate versal deformations of $L_{\infty}$ algebras with invariant inner products, which is related to the cyclic cohomology of these algebras. In the case of ordinary Lie algebras, only reductive algebras have invariant inner products, so such a deformation theory only applies to these cases. We note that among these, simple Lie algebras have no deformations, even as $L_{\infty}$ algebras. For super Lie algebras - and $L_{\infty}$ algebras in general - the picture is not so restricted.

\section{References}

[1] M. Alexandrov, M. Kontsevich, A. Schwarz, and O. Zaboronsky, The geometry of the master equation and topological quantum field theory, Internat. J. Modern Phys. A12 (1997), 1405-1423. 
[2] G. Barnich, R. Fulp, T. Lada, and J. Stasheff, The sh Lie structure of Poisson brackets in field theory, Comm. Math. Phys. 191 (1998), 585-601.

[3] F. A. Berends, G. J. H. Burgers and H. van Dam, Nuclear Physics B 260 (1985), 295-322.

[4] A. Fialowski, Deformations of Lie algebras, Math. USSR-Sb. 55 (1986), 467-473.

[5] A. Fialowski, An example of formal deformations of Lie algebras, in: NATO Conference on Deformation Theory of Algebras and Applications (Ciocco, 1986), Kluwer, 1988, 375-401.

[6] A. Fialowski and D. Fuchs, Singular deformations of Lie algebras on an example, in: Topics in Singularity Theory (Providence, RI), A. Varchenko and V. Vassilie (eds.), A.M.S. Transl. Ser. 2, 180, Amer. Math. Soc., 1997.

[7] A. Fialowski and D. Fuchs, Construction of miniversal deformations of Lie algebras, J. Funct. Anal. 161 (1999), 76-110.

[8] A. Fialowski and M. Penkava, Deformation theory of infinity algebras, preprint: math.RT/0101097, to appear in J. Algebra.

[9] A. Fialowski and G. Post, Versal deformation of the Lie algebra $\mathbf{L}_{\mathbf{2}}$, J. Algebra 236 (2001).

[10] M. Kontsevich, Feynman diagrams and low dimensional topology, in: First European Congress of Mathematics, Paris, 1992, Birkhäuser, Basel, 1994, 97-121.

[11] M. Kontsevich, Deformation quantization of Poisson manifolds, I, preprint q-alg/9709040, 1997.

[12] I. M. Krichever and S. P. Novikov, Algebras of Virasoro Type, Riemann surfaces and structures of the theory of solitons, Funct. Anal. Appl. 21 (1987), 126-142.

[13] T. Lada and M. Markl, Strongly homotopy Lie algebras, Comm. Algebra 23 (1995), 21472161.

[14] T. Lada and J. Stasheff, Introduction to sh Lie algebras for physicists, Intern. J. Theor. Phys. 32 (1993), 1087-1103, preprint hep-th 9209099.

[15] M. Markl, Cyclic operads and the homology of graph complexes, preprint Math/9801095, 1996.

[16] M. Penkava, $L_{\infty}$ algebras and their cohomology, preprint q-alg 9512014, 1996.

[17] M. Penkava, Infinity algebras, cohomology and cyclic cohomology, and infinitesimal deformations, preprint math.QA/0111088, 2001.

[18] M. Penkava, Infinity algebras and the homology of graph complexes, preprint q-alg 9601018, 1996.

[19] M. Penkava and A. Schwarz, $A_{\infty}$ algebras and the cohomology of moduli spaces, Dynkin Seminar, vol. 169, Amer. Math. Soc., 1995, 91-107.

[20] M. Penkava and L. Weldon, Infinity algebras, Massey products, and deformations, preprint math/9808058, 1996.

[21] M. Schlessinger and J. Stasheff, The Lie algebra structure of tangent cohomology and deformation theory, J. Pure Appl. Algebra 38 (1985), 313-322.

[22] M. Schlessinger and J. Stasheff, Deformation theory and rational homotopy type, to appear.

[23] J. D. Stasheff, On the homotopy associativity of H-spaces I, Trans. AMS 108 (1963), $275-292$.

[24] J. D. Stasheff, On the homotopy associativity of H-spaces II, Trans. AMS 108 (1963), 293-312.

[25] J. D. Stasheff, Closed string field theory, strong homotopy Lie algebras and the operad actions of moduli spaces, in: Conf. Proc. Lecture Notes Math. Phys., III, Internat. Press, Cambridge, MA, 1994, 265-288. 